\title{
Aging and the perception of local surface orientation from optical patterns of shading and specular highlights
}

\author{
J. Farley Norman ANd Elizabeth Y. WiesemanN \\ Western Kentucky University, Bowling Green, Kentucky
}

\begin{abstract}
Younger and older observers' ability to perceive local surface orientation from optical patterns of shading and specular highlights was investigated in two experiments. On each trial, the observers viewed a randomly generated, smoothly curved 3-D object and manipulated an adjustable gauge figure until its orientation matched that of a specific local region on the object's surface (cf. Koenderink, van Doorn, \& Kappers, 1992). The performance of both age groups was facilitated by the presence of binocular disparity (Experiment 1 ) and object rotation in depth (Experiment 2). Observers in both age groups were able to judge the surface tilt component of orientation more precisely than the slant component. Significant, but modest, effects of age were found in Experiment 1, but not in Experiment 2. The ability to perceive local surface orientation appears to be relatively well preserved with increasing age, at least through the age of 80 .
\end{abstract}

In everyday environments, solid objects are illuminated by a variety of light sources, both natural and artificial. The surface regions of these objects reflect varying amounts of light toward an observer's eyes, depending on their individual orientations. Surface regions that are perpendicular to the direction of illumination reflect the most light, whereas those that are parallel to the direction of illumination reflect no light. For those regions with intermediate orientations, the amount of light reflected is proportional to the cosine of the angle between the orientation of the surface normal and the direction facing the light source (Lambert's law). Leonardo da Vinci ( 1519/1970) was clearly aware of this relationship between surface orientation and the brightness of each region's reflected light. In his second book on light and shade, he stated that "the light which falls on a shaded body at the acutest angle receives the highest light, and the darkest portion is that which receives it at an obtuse angle" (p. 87). For objects with shiny surfaces, such as those composed of metal or glass, specular highlights are also visible to an observer; these smooth surfaces reflect light in a "mirror-like" manner, so that a reflection of the light source is visible on the surface of the object. The apparent position of the highlight depends not only on the position of the light and the surface geometry of the object, but also on the position of the observer.

As we have seen, the intensities of light reflected by an object vary across its surface. These optical patterns of shading and highlights contain a valuable amount of information about 3-D object shape. Artists have taken advantage of this fact for thousands of years, at least since the era of the Roman Empire (see, e.g., Gombrich, 1976). The use of chiaroscuro (i.e., light and shade) is especially important for painters, since many of the most perceptually important sources of information about 3-D shape, such as binocular disparity and motion parallax, cannot be depicted in a single static image of any kind. Over the past 600 years, artists such as van Eyck, Caravaggio, Vermeer, and de Hooch have used patterns of shading and specular highlights to effectively portray 3-D objects and scenes. In more recent times, vision researchers have thoroughly investigated how human observers use such patterns of shading and highlights to perceive various aspects of 3-D object shape (e.g., Bülthoff \& Mallot, 1988; Doorschot, Kappers, \& Koenderink, 2001; Fleming, Torralba, \& Adelson, 2004; Johnston \& Passmore, 1994; Koenderink, Kappers, Todd, Norman, \& Phillips, 1996; Koenderink, van Doorn, Christou, \& Lappin, 1996; Langer \& Bülthoff, 2000; Liu \& Todd, 2004; Norman, Todd, \& Orban, 2004; Norman, Todd, \& Phillips, 1995; Todd, Norman, Koenderink, \& Kappers, 1997; Todd \& Reichel, 1989). Given the extensive amount of research that has been conducted to date, it is surprising that no studies have yet examined how aging affects older observers' abilities to perceptually utilize optical patterns of shading and highlights. Aging is known to affect the ability of older observers to extract information about 3-D shape from random dot stereograms (Norman, Crabtree, et al., 2006; Norman, Dawson, \& Butler, 2000), patterns of motion parallax (Andersen \& Atchley, 1995; Norman, Clayton, Shular, \& Thompson, 2004), and kinetic depth effect displays (Norman et al., 2000). The primary purpose of our present set of experiments was to rectify this lack of information and evaluate the ability of older observers to utilize shading and 
highlights for the perception of 3-D object shape. Given the deterioration in older observers' ability to take advantage of traditional forms of binocular disparity and motion, they may rely even more heavily than younger observers upon other sources of 3-D information, such as shading and specular highlights. Thus, the ability to detect and utilize these sources of perceptual information may be relatively unaffected by increasing age. In the present set of experiments, we evaluated older observers' sensitivity to static patterns of shading and highlights (Experiments 1 and 2), binocularly disparate patterns of shading and highlights (Experiment 1), and the deforming patterns of shading and highlights that are obtained when shaded and highlighted object surfaces rotate in depth (Experiment 2).

Previous studies in which the effects of age upon the perception of 3-D shape have been evaluated have utilized sparsely sampled dotted surfaces (e.g., conventional random dot stereograms and kinetic depth effect displays; see Andersen \& Atchley, 1995; Norman, Clayton, et al., 2004; Norman, Crabtree, et al., 2006; Norman et al., 2000). Such studies are important because their stimuli contained only a single source of information about 3-D shape. It is thus possible, for example, to study whether and how increasing age affects the detection and utilization of binocular disparity independently of other sources of information, such as shading. It is important to note, however, that in ordinary environments, our behavior is targeted toward solid objects that are defined simultaneously by multiple sources of information: for example, reaching for and grasping a coffee mug, a baseball, or a stapler in a lighted room. The stimulus displays used in the present set of experiments possessed higher ecological validity than did those investigated previously, in that they depicted 3-D objects with solid surfaces and were thus potentially graspable (for research on aging and grasping solid objects, see Carnahan, Vandervoort, \& Swanson, 1998; Roy, Weir, Desjardins-Denault, \& Winchester, 1999).

Both Marr (1982, pp. 275-283) and Gibson (1950, pp. 92, 99) have proposed that human observers perceive the 3-D shape of environmental object surfaces in terms of local surface orientation - that is, that what we know best about an object's 3-D shape are its constituent surface orientations. The results of Norman and Todd (1996) and Norman, Todd, Norman, Clayton, and McBride (2006) are consistent with this idea; they found that human observers can discriminate the magnitudes of differences in surface orientation more precisely than they can the magnitudes of differences in either surface depth or surface curvature. At this point in time, no psychophysical studies have yet evaluated whether aging affects the accuracy and/or precision of observers' perceptions of local surface orientation. Therefore, an important secondary purpose of our study was to assess whether and to what extent aging affects the perception of local surface orientation.

\section{EXPERIMENT 1}

\section{Method}

Apparatus. The stimulus displays were created by a dualprocessor Apple Power Macintosh G4 computer (1.42 GHz) and were displayed on a 22-in. Mitsubishi Diamond Plus 200 color monitor. The rendering of the shaded surfaces was accelerated by a Radeon 9000 graphics accelerator (ATI Technologies). The stimuli were viewed by the observers from a distance of $100 \mathrm{~cm}$. Stereoscopic versions of the displays were presented to the observers using CrystalEyes2 LCD-shuttered glasses (StereoGraphics).

Stimulus displays. One hundred randomly shaped objects were created following the procedures used by Norman and Todd (1996, 1998), Norman et al. (1995), and Norman, Todd, and Orban (2004). Each object's surface was defined by the positions and orientations of 32,768 triangular polygons. Each object was depicted using some combination of texture, lambertian shading, and/or specular highlights. The surface shading was produced using a standard reflectance model (see, e.g., Foley, van Dam, Feiner, \& Hughes, 1996), in which the image intensities were determined by a combination of ambient, diffuse, and specular components. The ambient component was 0.3 for all surface types, except those surfaces defined only by specular highlights (for which the ambient component was zero). The diffuse component was 0.7 for surfaces defined by either lambertian shading or a combination of texture and lambertian shading. The diffuse component for the surfaces defined by shading and highlights was 0.4 , and the specular component was 0.3 . The highlights-only surfaces were created by setting the specular component to 1.0 and the diffuse component to 0.0 . The shininess exponent was 20 for all surfaces that possessed specular highlights. For those surfaces that contained texture, equal areas on the surfaces received equal amounts of texture. Examples of representative stimuli are illustrated in Figure 1.

Procedure. There were eight experimental conditions formed by the combination of two stereoscopic conditions (binocular views containing disparity and monocular views without disparity) and four surface types (shading and texture, shading and highlights, shading only, and specular highlights only). The observers completed 20 trials for each of the eight conditions across two experimental sessions ( 80 trials in each session, 160 trials total). One session was devoted to the stereoscopic (i.e., binocular) judgments, whereas the other session was devoted to the monocular judgments (the observers wore an eyepatch over one eye in the monocular conditions).
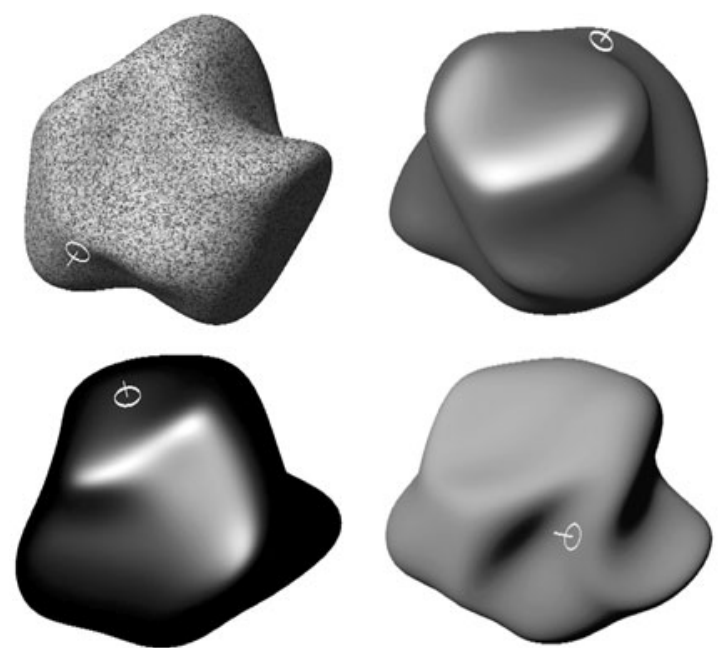

Figure 1. Examples of the types of surfaces and objects used in the experiments. Clockwise, starting from the upper left, are objects with surfaces defined by shading and texture, shading and highlights, shading only, and highlights only. A gauge figure is depicted on the surface of each object; the observers' task was to adjust the orientation of the circular part of the gauge figure in 3-D space until its orientation matched that of the local surface region directly underneath (when the circle possessed the same orientation as the underlying surface region, the "stick" part of the gauge figure was perpendicular to the surface). 
The observers' task on each trial was to adjust the orientation of a circular gauge figure (see, e.g., Koenderink, Kappers, et al., 1996; Koenderink, van Doorn, \& Kappers, 1992; Norman et al., 1995) until its orientation matched that of the surface region underneath it. Each surface region to be investigated was randomly chosen, subject only to the constraint that each region's actual slant was between $30^{\circ}$ and $70^{\circ}$. In order to maximize their performance, all the observers were given an unlimited amount of time to make their adjustments.

Prior to the start of the experimental trials, all the observers were familiarized with the task by having them complete a series of practice trials in which the adjustable gauge figure was placed onto various facets of randomly oriented stereoscopic cubes. These practice trials were continued until each observer was comfortable with the task; it also allowed us to ensure that the observers thoroughly understood the task before beginning the experimental trials.

Observers. Twenty-two observers participated in the experiment. One group of observers consisted of 11 older adults (mean age was 71.2 years, $S D=5.0$; the range of their ages was $65-81$ years). These observers were asked (i.e., self-report) whether they possessed eye or retinal problems, such as macular degeneration, glaucoma, or cataracts (none were reported). The other group consisted of 11 younger observers (mean age was 24.0 years, $S D=2.9$ ). The second author (E.Y.W.) participated in the experiment as one of the younger observers. Five of the younger observers viewed the stimulus displays directly, whereas the other 6 viewed the experimental stimuli with 0.5 neutral density filters (Kodak Wratten No. 96). Viewing the stimuli through the neutral density filters served to dim or reduce the brightnesses of these younger observers' retinal images by two thirds. It has been demonstrated that the retina of an average 60-year-old receives only one third of the light that the retina of a 20 -year-old would receive under identical viewing conditions (Weale, 1963, p. 168). In our present experiment, we thus made the younger observers' retinal images optically identical to those of much older adults. If there is an age effect and it is due to the reduction in the brightness of older observers' retinal images, we should be able to create a similar age effect in the younger observers by reducing the brightness of their retinal images. On the other hand, if the older adults perform more poorly than the younger observers who view the stimuli using 0.5 neutral density filters, this would suggest that the effect of age is due to neural, rather than optical, factors. The use of neutral density filters to simulate the optical effects of aging in younger observers has previously been used by other investigators (see, e.g., Bennett, Sekuler, \& Ozin, 1999; Elliott, Whitaker, \& MacVeigh, 1990; Habak \& Faubert, 2000; Sekuler \& Owsley, 1982).

All of the observers' acuities were measured at a distance of $100 \mathrm{~cm}$, using a Landolt C chart (Riggs, 1965). The younger observers' average acuity was $0.98 \mathrm{~min}^{-1}$ (for both young groups), whereas that for the older observers was slightly less, $0.97 \mathrm{~min}^{-1}$ ( $1.0 \mathrm{~min}^{-1}$ is equivalent to $20 / 20$ vision measured at $20 \mathrm{ft} ; 0.8 \mathrm{~min}^{-1}$ is equivalent to $20 / 25$ vision). If the observers typically wore corrective lenses (e.g., bifocals), they used the correction that gave the best visual acuity for viewing the experimental stimuli. All of the observers (except E.Y.W.) were naive with regard to the purposes of the experiment and were unaware of how the experimental stimuli had been generated.

Prior to the start of the experimental trials, all the observers' stereoscopic capabilities were tested by showing them random dot stereograms (Julesz, 1964, 1971). According to Julesz (1971), "random-dot stereograms yield an objective, unfakable test for stereopsis" (p. 276). All of the observers in our experiment (both younger and older) were able to spontaneously perceive and describe the depth and 3-D shape of surfaces depicted in random dot stereograms (maximum disparity was $10.3 \mathrm{~min}$ arc) and, thus, possessed good stereopsis.

\section{Results and Discussion}

The results are shown in Figures 2-6. In the following analyses, the results for the two groups of younger observers (who viewed the displays with and without the 0.5 neutral density filters) were combined, since there was no significant difference between them ${ }^{1}[F(1,9)=0.015, p=.905]$. The left panel of Figure 2 plots the magnitude of the observers' total errors (i.e., the difference or angle between the actual surface orientations and the observers' judged surface orientations) for both the binocular and the monocular conditions (conditions with and without binocular disparity, respectively). The right panel of Figure 2 plots the observers' performance as a function of the various surface types. The main effects of both stereoscopic viewing (left panel) and surface type (right panel) were significant $[F(1,20)=$ $22.7, M S_{\mathrm{e}}=44.6, p=.0001, \eta^{2}=.53$, and $F(3,60)=6.9$, $M S_{\mathrm{e}}=16.0, p<.001, \eta^{2}=.26$, respectively].

In addition to the main effects of stereoscopic viewing and surface type, there was a significant main effect of age $\left[F(1,20)=4.43, M S_{\mathrm{e}}=195.2, p<.05, \eta^{2}=.18\right]$. This effect of age is also readily apparent in the results shown in Figure 2. The average error magnitudes produced by the younger and older observers were $24.7^{\circ}$ and $29.1^{\circ}$, respectively; the older observers' errors were $17.8 \%$ higher than those exhibited by the younger observers. However, the age $\times$ stereoscopic view and age $\times$ surface type interactions were not significant $[F(1,20)=0.098, p=.76$, and $F(3,60)=0.02, p=.996$, respectively]. The older observers' judgments were, therefore, also facilitated by the presence of binocular disparity (see the left panel of Figure 2), and their judgments were also most accurate for the surfaces defined by shading and texture (see the right panel of Figure 2).

Figure 3 illustrates a significant stereo $\times$ surface type interaction $\left[F(3,60)=6.4, M S_{\mathrm{e}}=10.4, p<.001, \eta^{2}=\right.$ .24]. It is readily apparent that the improvement (i.e., decrease in error) due to the introduction of binocular disparity was larger for the textured surfaces $\left(8.2^{\circ}\right)$ than for the surfaces defined only by shading and specular highlights $\left(4.6^{\circ}\right)$. The improvements due to binocular disparity were substantial: There was a $28.7 \%$ decrease in error for the textured surfaces and a $15.4 \%$ decrease in error for the shaded and highlight-defined surfaces. The fact that the decrease in error was larger for the textured surfaces is responsible for the observed interaction.

The magnitude of the stereo $\times$ surface type interaction illustrated in Figure 3 was itself influenced by age [i.e., the age $\times$ stereo $\times$ surface type interaction was significant; $\left.F(3,60)=2.8, M S_{\mathrm{e}}=10.4, p<.05, \eta^{2}=.13\right]$. The relevant results for the younger and older observers are presented individually in Figures 4 and 5, respectively. It is readily apparent from a comparison of Figures 4 and 5 that the younger and older observers exhibited the same qualitative pattern of results. For example, the observers in both age groups performed best (produced the lowest errors) for surfaces defined by binocular disparity, with the largest improvements occurring for the surfaces depicted with both shading and texture. For those surfaces, the magnitudes of the errors obtained by the younger observers for monocular viewing decreased by $36.0 \%$ when binocular disparity was added; the analogous decrease for the older observers was a smaller $22.1 \%$. The younger observers' errors for monocularly viewed surfaces defined by shading and highlights decreased by $21.6 \%$ when binocular 

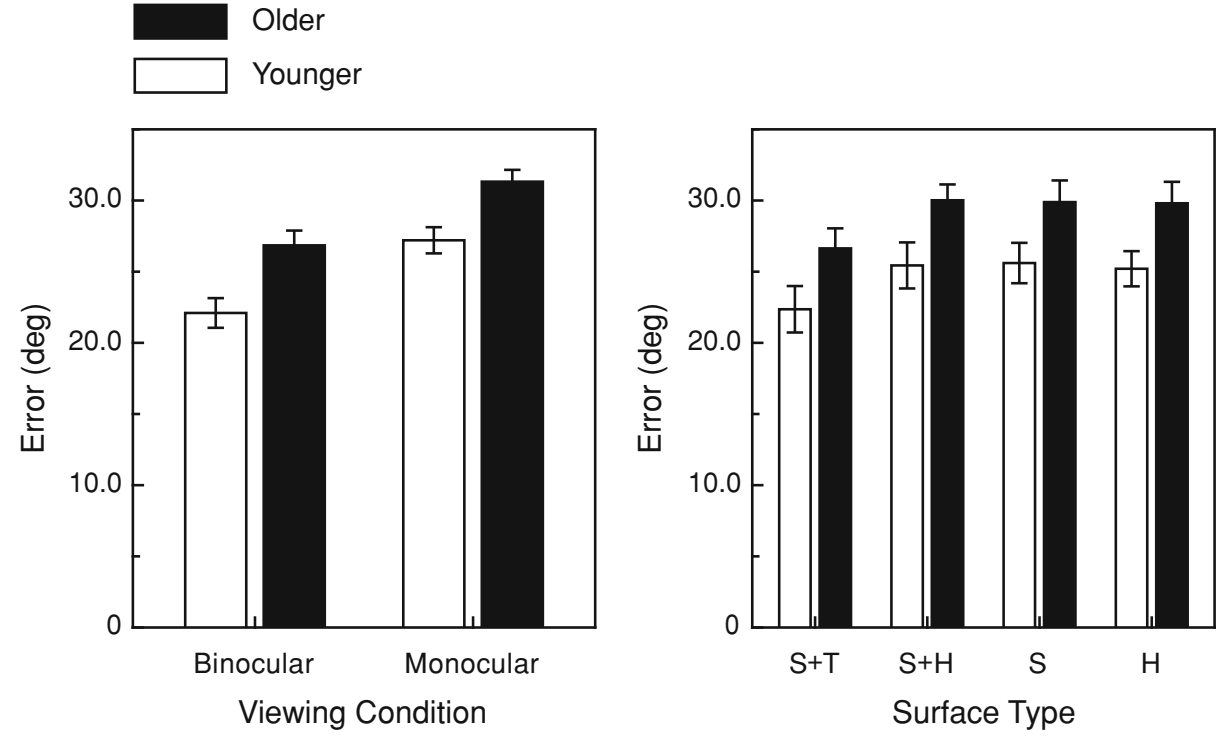

Figure 2. Results of Experiment 1 for the younger and older observers. The total magnitudes of the observers' errors (i.e., between the actual surface orientations and the judged surface orientations) are plotted as functions of the presence or absence of binocular disparity (left panel) and surface type (right panel). " $S+T$ " refers to the surfaces defined by shading and texture. " $S+H$ " refers to the surfaces defined by shading and specular highlights. " $S$ " and " $H$ " refer to surfaces defined only by shading and only by specular highlights, respectively. The error bars indicate $\pm 1 S E$.

disparity was added; the corresponding improvement in performance was a smaller $9.7 \%$ for the older observers. These quantitative differences generated the significant age $\times$ stereo $\times$ surface type interaction.

Figure 6 decomposes the total error and plots two representative observers' (27 and 79 years of age) adjusted orientations in terms of surface slant and tilt. Slant and tilt are two components of surface orientation (see, e.g., Koenderink, 1986; Stevens, 1983). Slant is defined as the angle between any given region's surface normal and a vector pointing toward an observer's line of sight, and tilt refers to the direction in the projected image in which the

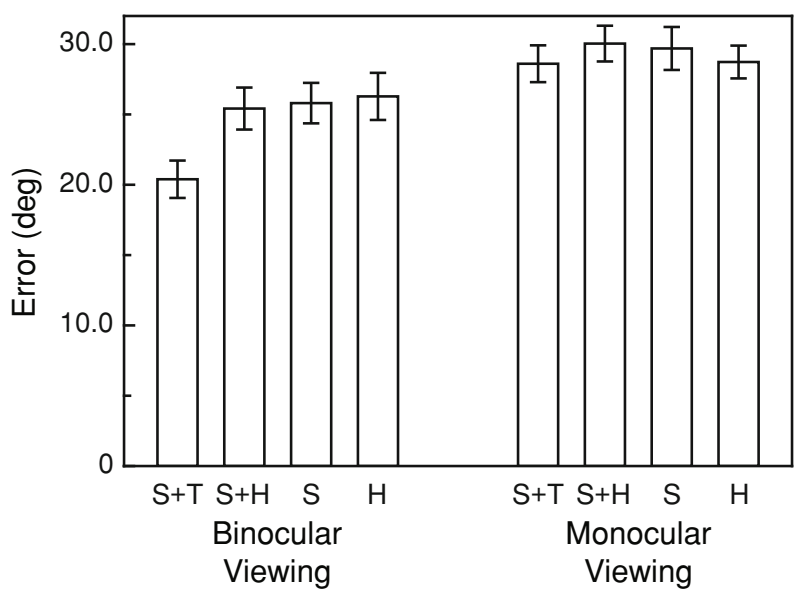

Figure 3. Results of Experiment 1. An illustration of the significant stereo (binocular disparity vs. no binocular disparity) $\times$ surface type interaction. The error bars indicate $\pm 1 S E$. slant occurs. Possible values for slant (for visible surface regions) thus range from $0^{\circ}$ to $90^{\circ}$, whereas possible values for tilt range from $0^{\circ}$ to $360^{\circ}$. As can be seen from these results, the younger and older observers were able to judge surface tilt much more precisely than surface slant. The average Pearson $r$ correlation magnitudes for the younger observers were .46 and .93 for surface slant and tilt, respectively. The analogous correlations for the older observers were .39 and .89 , respectively. These values indicate that although human observers can accurately judge the direction in which a given surface region slants in depth (e.g., the surface slants to the left, slants to the right, slants at a $20^{\circ}$ angle from vertical, etc.), they are less able to judge the magnitude of slant-in-depth.

\section{EXPERIMENT 2}

In Experiment 1, the role of binocular disparity was investigated, and it was found that the presence of binocular disparity (i.e., stereoscopic viewing) facilitated the judgments of both the older and the younger observers (see Figure 2). The purpose of Experiment 2 was to similarly evaluate the extent to which older observers can utilize motion to improve their ability to perceive local surface orientation from optical patterns containing shading and specular highlights.

\section{Method}

Apparatus. The apparatus was identical to that used in Experiment 1.

Stimulus displays. The stimulus displays were identical in almost all aspects to those used in the shading-only and highlightsonly conditions in Experiment 1. In this experiment, no binocular 


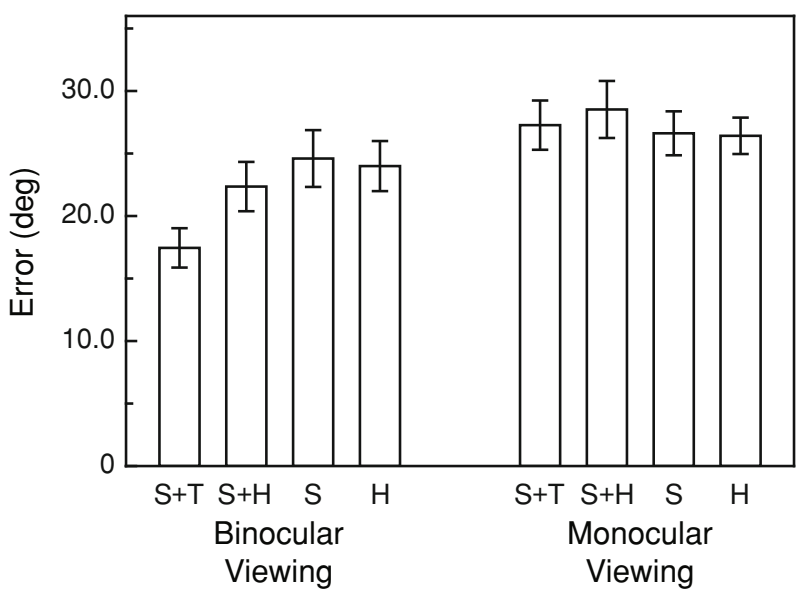

Figure 4. Results for all eight conditions in Experiment 1 for the younger observers. The error bars indicate $\pm 1 S E$.

disparity was present. The stimuli were thus identical to those used in the monocular conditions in Experiment 1, with one exception: In Experiment 2, the objects in half of the experimental conditions were depicted in motion (i.e., rotation in depth about a Cartesian vertical axis). For those conditions incorporating motion, each object oscillated in depth $\pm 12^{\circ}$ from a randomly chosen home orientation. The apparent motion sequences consisted of 20 individual frames; the objects rotated $1.2^{\circ}$ at each frame transition. An example of a typical apparent motion sequence is presented in Figure 7. Each individual frame was updated at a rate of $18.75 \mathrm{~Hz}$.

Procedure. The observers' task was the same as that used in Experiment 1: to adjust the orientation of a circular gauge figure so that its orientation matched that of a randomly chosen underlying surface region. Once again, the observers were given an unlimited amount of time to perform the task. The observers made 20 judgments for each of the four experimental conditions in a single session (i.e., 80 trials).

Observers. Ten younger observers (mean age was 24.4 years, $S D=3.2$ ) and 10 older observers (mean age was 71.8 years, $S D=$ 5.3 ; the range of their ages was $65-81$ years) participated in the experiment. Nine of the older observers and 8 of the younger observers (including E.Y.W.) had previously participated in Experiment 1. The younger observers' average acuity was $1.0 \mathrm{~min}^{-1}$, and that for

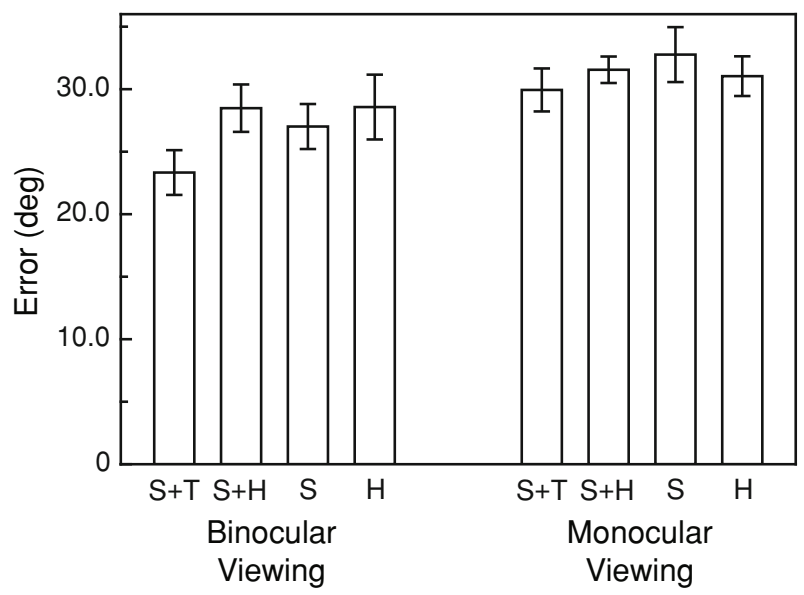

Figure 5. Results for all eight conditions in Experiment 1 for the older observers. The error bars indicate $\pm 1 S E$. the older observers was slightly less, $0.94 \mathrm{~min}^{-1}$. No observer reported any significant eye disorders, such as macular degeneration, glaucoma, and so forth. All of the observers (except E.Y.W.) were naive with regard to the purposes of the experiment, the nature of the experimental stimuli, and so forth.

\section{Results and Discussion}

The results are shown in Figure 8. The right panel illustrates a significant effect of surface type $[F(1,18)=14.4$, $\left.M S_{\mathrm{e}}=16.2, p<.002, \eta^{2}=.45\right]$. The observers' ability to estimate orientation was better (i.e., lower error) when they viewed surfaces defined by lambertian shading than when they viewed similar surfaces defined only by specular highlights. The observers' performance for both of these conditions was facilitated, however, by the presence of motion $\left[F(1,18)=82.1, M S_{\mathrm{e}}=7.5, p<.0001, \eta^{2}=\right.$ $.82]$. This significant effect of motion is depicted in the left panel of Figure 8. The magnitudes of the observers' errors decreased by an average of $18.7 \%$ when the object surfaces rotated in depth. Unlike in Experiment 1, there was no significant effect of age [the average error magnitudes of the younger and older observers were $25.3^{\circ}$ and $28.6^{\circ}$, respectively; $F(1,18)=1.6, p=.22$ ]. It is possible that with large enough samples, this small quantitative difference between the performances of the two age groups could become statistically significant. A power analysis revealed that we would need a sample of approximately 100 observers ( 50 younger and 50 older observers) to have an $80 \%$ chance of detecting a significant effect of age as small as the difference that was obtained in the present experiment.

In previous studies concerning aging and the perception of 3-D shape from motion (Andersen \& Atchley, 1995; Norman, Clayton, et al., 2004; Norman et al., 2000), dotted and sparsely sampled surfaces were used as stimuli. Interpolation processes would, therefore, be required in order for the observers to perceive smooth surfaces (i.e., to "complete" the surfaces by filling in the gaps between the points). Significant effects of age were found in these prior experiments, especially when the temporal correspondences of the points were disrupted (e.g., Norman, Clayton, et al., 2004; Norman et al., 2000). In the present experiment, the observers viewed 3-D object surfaces that were much more realistic (i.e., they were solid, and therefore, interpolation processes were not required) and were depicted with smooth shading or specular highlights (simulating either matte or shiny surfaces, respectively). A significant effect of age was not found in the present experiment. It is possible that the relatively good performance of the older observers was due to the more realistic nature of the stimulus displays (i.e., more information was available to support the observers' judgments than in the prior studies). Another explanation may involve the nature of the task. The previous experiments (e.g., Norman, Clayton, et al., 2004; Norman et al., 2000) required the observers to discriminate between the 3-D shapes of objects presented across different trials (thus, the discrimination task had a memory component), whereas the task used in the present experiment required the older observers only to judge the local shape of a single object presented on any 


\section{Observer 12} Age $=79$
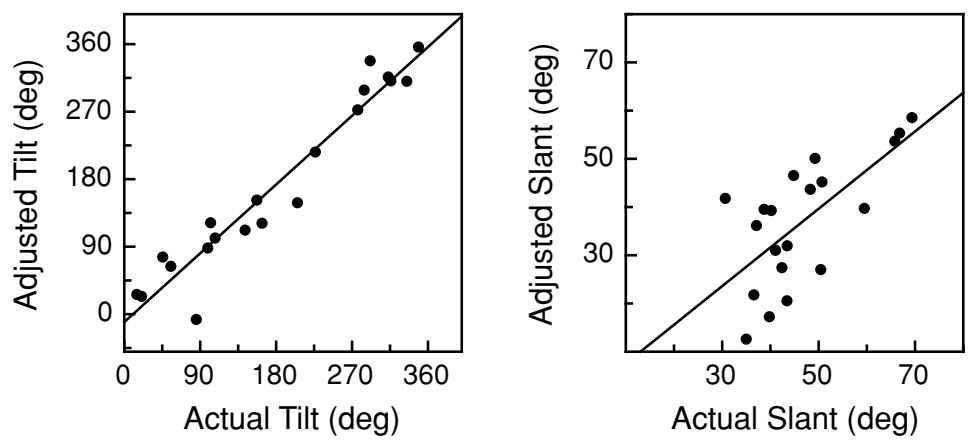

Observer 14 Age $=27$
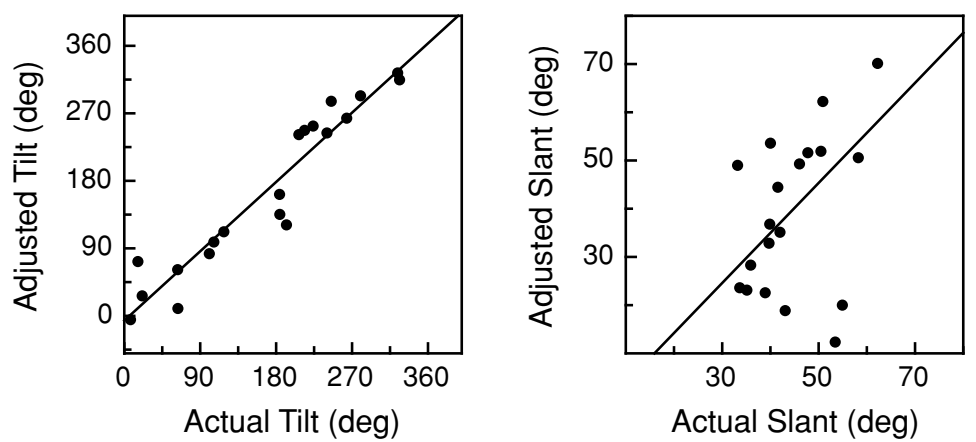

Figure 6. Results of Experiment 1 illustrating the difference in precision in the observers' judgments of surface slant and tilt. The top row presents the results of a representative older observer for the condition in which the surfaces were defined by stereoscopic patterns of shading and highlights. The bottom row presents the analogous results for a representative younger observer. Note that although there is a 52-year difference in their ages, these observers' results are remarkably similar.

given trial (thus, the memory demands of the present task were much lower).

\section{GENERAL DISCUSSION}

The results of Experiment 1 support previous findings of age-related deficits in the stereoscopic perception of 3-D shape (Norman et al., 2000; Norman, Crabtree, et al., 2006), as well as the findings in those studies that have found reductions in stereoacuity with increasing age (Bell, Wolf, \& Bernholz, 1972; Brown, Yap, \& Fan, 1993; Haegerstrom-Portnoy, Schneck, \& Brabyn, 1999; Jani, 1966; Wright \& Wormald, 1992). It is interesting to note that whereas all of the studies to date in which the stereoscopic perception of 3-D shape has been evaluated have shown significant effects of age, the studies in which stereoacuity and aging have been evaluated have had mixed and contradictory results. For example, whereas many studies have shown that stereoacuity declines with age (Bell et al., 1972; Brown et al., 1993; HaegerstromPortnoy et al., 1999; Jani, 1966; Wright \& Wormald, 1992), many other, similar studies have shown no deterioration in stereoacuity with increases in age (Greene \& Madden, 1987; Hofstetter \& Bertsch, 1976; Tiffin, 1952; Yekta, Pickwell, \& Jenkins, 1989).

The results of Experiment 1 did reveal that there was a significant effect of age upon the observers' ability to judge local surface orientation for all the surface types. It is also true, however, that the effect of age was rela- tively small and quantitative; in almost every other way, the pattern of the older observers' results was qualitatively similar to that of the younger observers (see Figures 2-6). For example, the older observers' performance was facilitated by both the presence of binocular disparity (Experiment 1) and object rotation in depth (Experiment 2). The results would also appear to indicate that older observers can effectively perceive local surface orientation from optical patterns of shading and specular highlights and that they can do so with almost the same level of accuracy and precision as observers who are $40-50$ years younger. In addition, the observers in both age groups were much more precise at judging surface tilt than surface slant (Figure 6).

The results of Experiment 1 revealed that the observers' ability to perceive local surface orientation improved when they viewed the surfaces stereoscopically. This improvement occurred not only for the textured condition, but also for the conditions in which the surfaces lacked visible texture and were defined exclusively by image shading and specular highlights. This is important because, when surface texture is present, it can serve as a carrier for traditional forms of binocular disparity. Binocular disparity is conventionally defined as the difference in the projected positions of identifiable surface features across an observer's left and right eyes. When texture is present, the individual texture elements mark and identify individual surface positions that then can be matched across the left and right eyes' views. Such tradi- 


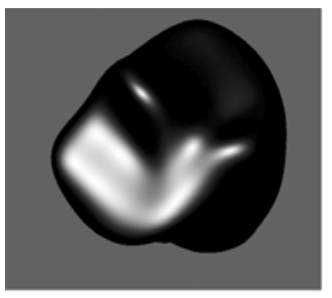

13

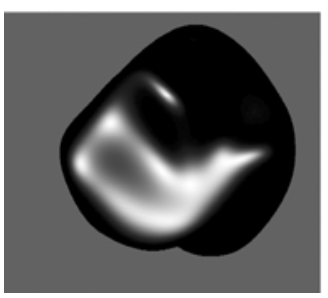

5

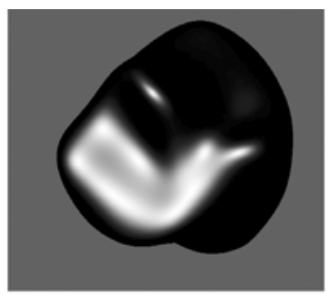

17

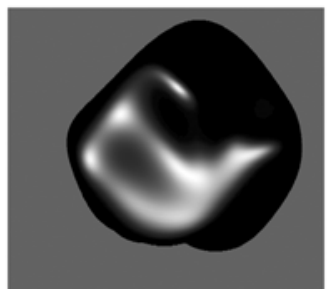

9

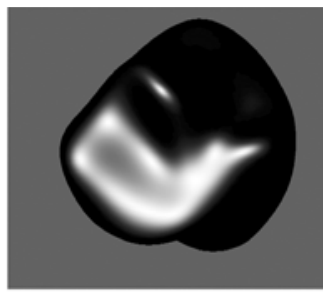

20

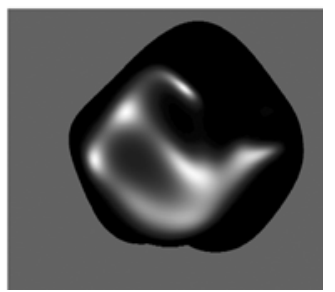

Figure 7. Six frames (Frames 1, 5, 9, 13, 17, and 20) taken from a representative apparent motion sequence. The depicted object's surface was shiny and was optically defined by specular highlights. Note that the rotation of the object in depth produced a complex deformation of the pattern of specular highlights.

tional binocular disparities do not occur when observers view stereoscopic surfaces defined only by shading and/or specular highlights. For these types of surfaces, only patterns of smoothly changing light intensities are reflected into the observers' eyes; there are no marks or features that can identify corresponding surface locations across an observer's left and right retinal images. Nevertheless, the observers in our Experiment 1 (both younger and older) were able to take advantage of the information present in the binocular shaded and highlight conditions: The observers' errors decreased an average of $12.3 \%$ when they stereoscopically viewed the shaded and highlight-defined surfaces (see Figures 3-5; the decrease was $11.8 \%$ for the older observers and $12.8 \%$ for the younger observers). These results confirm and extend earlier psychophysical research in which binocularly disparate patterns of shading and highlights have been investigated (see, e.g., Bülthoff \& Mallot, 1988; Doorschot et al., 2001; Johnston \& Passmore, 1994; Norman, Todd, \& Orban, 2004; Norman et al., 1995). In a similar vein, surface texture, when available, serves as a carrier to define the velocities that are needed for conventional structure-from-motion processes. Note that in Experiment 2, in which neither of the surface types contained texture, the observers greatly benefited
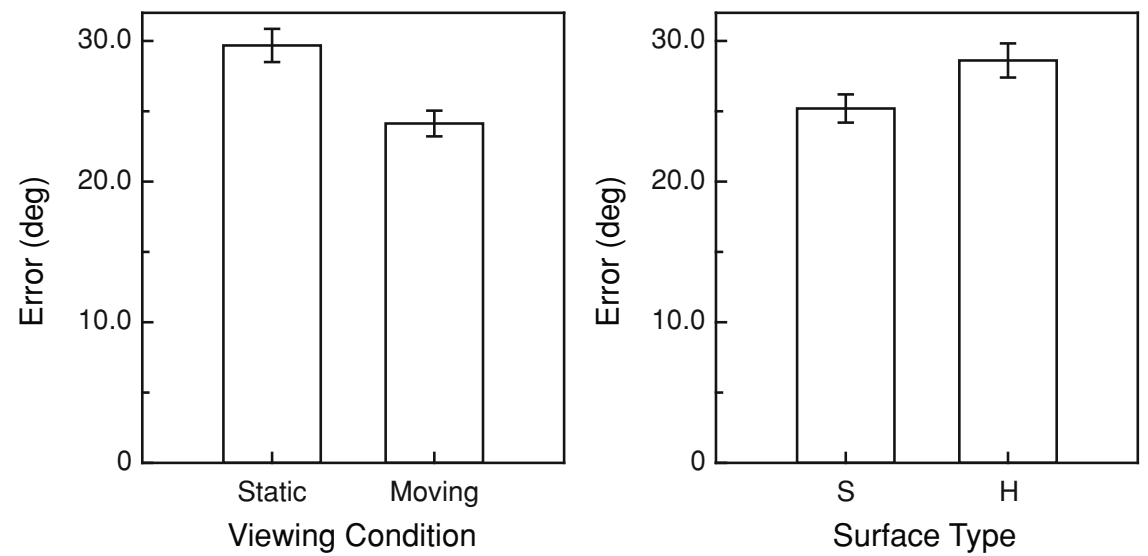

Figure 8. Results of Experiment 2 for all the observers. The total magnitudes of the observers' errors (i.e., between the actual surface orientation and the judged surface orientation) are plotted as functions of the presence or absence of motion (left panel) and surface type (right panel; $\mathrm{S}$, shading; $\mathrm{H}$, highlights). The error bars indicate $\pm 1 S E$. 
from the object motion (see Figure 8). This rotation of the objects in depth produced deformations in the resulting patterns of smooth shading and specular highlights (see Figure 7 for an example deformation). Even though these deformations of shading and highlights cannot be successfully analyzed using conventional structure-from-motion algorithms (see Norman \& Todd, 1994; Norman et al., 1995; Todd, 1985), our observers were able to utilize these deformations to more accurately perceive the local shape of the object surfaces. The results of our Experiment 2 are similar to those in a number of past investigations (Koenderink, Kappers, et al., 1996; Norman, Todd, \& Orban, 2004; Norman et al., 1995) but also extend them to a considerable degree by documenting that older observers can also take advantage of and use deforming shading and highlight fields to effectively perceive 3-D object shape. The results of Experiments 1 and 2 suggest that the ability to perceive local surface orientation from optical patterns of shading and highlights is relatively robust and continues to function effectively at least through the age of 80 .

\section{AUTHOR NOTE}

Correspondence concerning this article should be addressed to J. F. Norman, Department of Psychology, Western Kentucky University, 1906 College Heights Blvd. \#21030, Bowling Green, KY 42101-1030 (e-mail: farley.norman@wku.edu).

\section{REFERENCES}

Andersen, G. J., \& Atchley, P. (1995). Age-related differences in the detection of three-dimensional surfaces from optic flow. Psychology \& Aging, 10, 650-658.

BeLl, B., Wolf, E., \& Bernholz, C. D. (1972). Depth perception as a function of age. Aging \& Human Development, 3, 77-81.

Bennett, P. J., Sekuler, A. B., \& Ozin, L. (1999). Effects of ageing on calculation efficiency and equivalent noise. Journal of the Optical Society of America A, 16, 654-668.

BROWN, B., YAP, M. K. H., \& FAN, W. C. S. (1993). Decrease in stereoacuity in the seventh decade of life. Ophthalmic \& Physiological Optics, 13, 138-142.

BüLthOFF, H. H., \& MALlot, H. A. (1988). Integration of depth modules: Stereo and shading. Journal of the Optical Society of America A, 5, 1749-1758.

Carnahan, H., Vandervoort, A. A., \& Swanson, L. R. (1998). The influence of aging and target motion on the control of prehension. Experimental Aging Research, 24, 289-306.

DA VINCI, L. (1970). Second book on light and shade. In J. P. Richter (Ed.), The notebooks of Leonardo da Vinci (Vol. 1, pp. 87-90). New York: Dover. (Original work published approximately 1519)

Doorschot, P. C. A., Kappers, A. M. L., \& KoenderinK, J. J. (2001). The combined influence of binocular disparity and shading on pictorial shape. Perception \& Psychophysics, 63, 1038-1047.

Elliott, D., WhitaKer, D., \& MacVeigh, D. (1990). Neural contribution to spatiotemporal contrast sensitivity decline in healthy ageing eyes. Vision Research, 30, 541-547.

Fleming, R. W., Torralba, A., \& ADElson, E. H. (2004). Specular reflections and the perception of shape. Journal of Vision, 4, 798-820.

Foley, J. D., van Dam, A., Feiner, S. K., \& Hughes, J. F. (1996). Computer graphics: Principles and practice (2nd ed. in C). Reading, MA: Addison-Wesley.

GiBson, J. J. (1950). The perception of the visual world. Boston: Houghton Mifflin.

Gombrich, E. H. (1976). The heritage of Apelles: Studies in the art of the Renaissance. Ithaca, NY: Cornell University Press.

Greene, H. A., \& MADDEN, D. J. (1987). Adult age differences in visual acuity, stereopsis, and contrast sensitivity. American Journal of $\mathrm{Op}$ tometry \& Physiological Optics, 64, 749-753.
HABAK, C., \& FAUBERT, J. (2000). Larger effect of aging on the perception of higher-order stimuli. Vision Research, 40, 943-950.

Haegerstrom-Portnoy, G., SCHNeCK, M. E., \& Brabyn, J. A. (1999). Seeing into old age: Vision function beyond acuity. Optometry \& Vision Science, 76, 141-158.

HofstetTer, H. W., \& BerTsCh, J. D. (1976). Does stereopsis change with age? American Journal of Optometry \& Physiological Optics, 53, 664-667.

JANI, S. N. (1966). The age factor in stereopsis screening. American Journal of Optometry \& Archives of the American Academy of Optometry, 43, 653-657.

Johnston, A., \& PAssmore, P. J. (1994). Independent encoding of surface orientation and surface curvature. Vision Research, 34, 3005-3012.

Julesz, B. (1964). Binocular depth perception without familiarity cues. Science, 145, 356-362.

JuLESZ, B. (1971). Foundations of cyclopean perception. Chicago: University of Chicago Press.

KoenderinK, J. J. (1986). Optic flow. Vision Research, 26, 161-180.

Koenderink, J. J., Kappers, A. M. L., TodD, J. T., Norman, J. F., \& Phillips, F. (1996). Surface range and attitude probing in stereoscopically presented dynamic scenes. Journal of Experimental Psychology: Human Perception \& Performance, 22, 869-878.

KoEnderink, J. J., VAN DoORn, A. J., Christou, C., \& Lappin, J. S. (1996). Perturbation study of shading in pictures. Perception, 25, 1009-1026.

KoEnderink, J. J., van Doorn, A. J., \& Kappers, A. M. L. (1992). Surface perception in pictures. Perception \& Psychophysics, 52, 487-496.

LANGER, M. S., \& Bülthoff, H. H. (2000). Depth discrimination from shading under diffuse lighting. Perception, 29, 649-660.

LiU, B., \& TodD, J. T. (2004). Perceptual biases in the interpretation of 3D shape from shading. Vision Research, 44, 2135-2145.

MARR, D. (1982). Vision: A computational investigation into the human representation and processing of visual information. San Francisco: Freeman.

Norman, J. F., Clayton, A. M., Shular, C. F., \& Thompson, S. R. (2004). Aging and the perception of depth and 3-D shape from motion parallax. Psychology \& Aging, 19, 506-514.

Norman, J. F., Crabtree, C. E., Herrmann, M., Thompson, S. R., Shular, C. F., \& Clayton, A. M. (2006). Aging and the perception of 3-D shape from dynamic patterns of binocular disparity. Perception \& Psychophysics, 68, 94-101.

NoRman, J. F., Dawson, T. E., \& Butler, A. K. (2000). The effects of age upon the perception of depth and 3-D shape from differential motion and binocular disparity. Perception, 29, 1335-1359.

Norman, J. F., \& TodD, J. T. (1994). Perception of rigid motion in depth from the optical deformations of shadows and occlusion boundaries. Journal of Experimental Psychology: Human Perception \& Performance, 20, 343-356.

NORMAN, J. F., \& TODD, J. T. (1996). The discriminability of local surface structure. Perception, 25, 381-398.

Norman, J. F., \& ToDD, J. T. (1998). Stereoscopic discrimination of interval and ordinal depth relations on smooth surfaces and in empty space. Perception, 27, 257-272.

Norman, J. F., Todd, J. T., Norman, H. F., Clayton, A. M., \& McBRIDE, T. R. (2006). Visual discrimination of local surface structure: Slant, tilt, and curvedness. Vision Research, 46, 1057-1069.

Norman, J. F., Todd, J. T., \& Orban, G. A. (2004). Perception of threedimensional shape from specular highlights, deformations of shading, and other types of visual information. Psychological Science, 15, 565-570.

Norman, J. F., TodD, J. T., \& Phillips, F. (1995). The perception of surface orientation from multiple sources of optical information. Perception \& Psychophysics, 57, 629-636.

RIGGS, L. A. (1965). Visual acuity. In C. H. Graham (Ed.), Vision and visual perception (pp. 321-349). New York: Wiley.

Roy, E. A., Weir, P. L., Desjardins-Denault, S., \& Winchester, T. (1999). Pointing versus grasping in young and older adults. Developmental Neuropsychology, 16, 19-27.

SEKUlER, R., \& OwSLEY, C. (1982). The spatial vision of older humans. In R. Sekuler, D. Kline, \& K. Dismukes (Eds.), Aging and human visual function (pp. 185-202). New York: Liss. 
Stevens, K. (1983). Slant-tilt: The visual encoding of surface orientation. Biological Cybernetics, 46, 183-195.

Tiffin, J. (1952). Industrial psychology. New York: Prentice Hall.

ToDD, J. T. (1985). Perception of structure from motion: Is projective correspondence of moving elements a necessary condition? Journal of Experimental Psychology: Human Perception \& Performance, 11, 689-710.

ToDD, J. T., Norman, J. F., KoEnderinK, J. J., \& KaPPERS, A. M. L. (1997). Effects of texture, illumination, and surface reflectance on stereoscopic shape perception. Perception, 26, 807-822.

TodD, J. T., \& ReICHEL, F. D. (1989). Ordinal structure in the visual perception and cognition of smoothly curved surfaces. Psychological Review, 96, 643-657.

WEALE, R. A. (1963). The aging eye. New York: Lewis.

Wright, L. A., \& Wormald, R. P. L. (1992). Stereopsis and ageing. Eye, 6, 473-476.

Yekta, A. A., Pickwell, L. D., \& Jenkins, T. C. A. (1989). Binocular vision, age and symptoms. Ophthalmic \& Physiological Optics, 9, $115-120$.

\section{NOTE}

1. Previous studies have similarly shown that younger observers' performance is not adversely affected when their retinal illuminance is reduced by neutral density filters to match the lowered retinal illuminance that normally exists for older observers because of lens opacification or senile miosis (see Bennett, Sekuler, \& Ozin, 1999, in which little or no effect of reduced retinal illumination for high-noise conditions was shown; see also Elliott, Whitaker, \& MacVeigh, 1990; Habak \& Faubert, 2000; Norman, Clayton, Shular, \& Thompson, 2004; Norman, Crabtree, et al., 2006). The present results and those of the previous studies suggest that the various effects of age that have been obtained are not due to optical factors but, rather, to neurophysiological changes within the visual system.

(Manuscript received June 29, 2005;

revision accepted for publication February 20, 2006.) 